\title{
Dicycle Cover of Hamiltonian Oriented Graphs
}

\author{
Khalid A. Alsatami, ${ }^{1}$ Hong-Jian Lai, ${ }^{2}$ and Xindong Zhang ${ }^{3}$ \\ ${ }^{1}$ Department of Mathematics, College of Science, Qassim University, P.O. Box 6644, Buraydah 51452, Saudi Arabia \\ ${ }^{2}$ Department of Mathematics, West Virginia University, Morgantown, WV 26506, USA \\ ${ }^{3}$ College of Mathematics Sciences, Xinjiang Normal University, Urumqi 830054, China
}

Correspondence should be addressed to Khalid A. Alsatami; kaf043@gmail.com

Received 9 October 2015; Accepted 31 December 2015

Academic Editor: Kinkar Ch Das

Copyright (C) 2016 Khalid A. Alsatami et al. This is an open access article distributed under the Creative Commons Attribution License, which permits unrestricted use, distribution, and reproduction in any medium, provided the original work is properly cited.

A dicycle cover of a digraph $D$ is a family $\mathscr{F}$ of dicycles of $D$ such that each $\operatorname{arc}$ of $D$ lies in at least one dicycle in $\mathscr{F}$. We investigate the problem of determining the upper bounds for the minimum number of dicycles which cover all arcs in a strong digraph. Best possible upper bounds of dicycle covers are obtained in a number of classes of digraphs including strong tournaments, Hamiltonian oriented graphs, Hamiltonian oriented complete bipartite graphs, and families of possibly non-Hamiltonian digraphs obtained from these digraphs via a sequence of 2 -sum operations.

\section{The Problem}

We consider finite loopless graphs and digraphs, and undefined notations and terms will follow [1] for graphs and [2] for digraphs. In particular, a cycle is a 2-regular connected nontrivial graph. A cycle cover of a graph $G$ is a collection $\mathscr{C}$ of cycles of $G$ such that $E(G)=\bigcup_{C \in \mathscr{C}} E(C)$. Bondy [3] conjectured that if $G$ is a 2 -connected simple graph with $n \geq 3$ vertices, then $G$ has a cycle cover $\mathscr{C}$ with $|\mathscr{C}| \leq(2 n-3) / 3$. Bondy [3] showed that this conjecture, if proved, would be best possible. Luo and Chen [4] proved that this conjecture holds for 2-connected simple cubic graphs. It has been shown that, for plane triangulations, serial-parallel graphs, or planar graphs in general, one can have a better bound for the number of cycles used in a cover [5-8]. Barnette [9] proved that if $G$ is a 3-connected simple planar graph of order $n$, then the edges of $G$ can be covered by at most $(n+1) / 2$ cycles. Fan [10] settled this conjecture by showing that it holds for all simple 2connected graphs. The best possible number of cycles needed to cover cubic graphs has been obtained in $[11,12]$.

A directed path in a digraph $D$ from a vertex $u$ to a vertex $v$ is called a $(u, v)$-dipath. To emphasize the distinction between graphs and digraphs, a directed cycle or path in a digraph is often referred to as a dicycle or dipath. It is natural to consider the number of dicycles needed to cover a digraph. Following [2], for a digraph $D, V(D)$ and $A(D)$ denote the vertex set and arc set of $D$, respectively. If $A^{\prime} \subseteq A(D)$, then $D\left[A^{\prime}\right]$ is the subdigraph induced by $A^{\prime}$. Let $K_{n}^{*}$ denote the complete digraph on $n$ vertices. Any simple digraph $D$ on $n$ vertices can be viewed as a subdigraph of $K_{n}^{*}$. If $W$ is an arc subset of $A\left(K_{n}^{*}\right)$, then $D+W$ denotes the digraph $K_{n}^{*}[A(D) \cup W]$.

A digraph $D$ is strong if, for any distinct $u, v \in V(D), D$ has a $(u, v)$-dipath. As in [2], $\lambda(D)$ denotes the arc-strongconnectivity of $D$. Thus a digraph $D$ is strong if and only if $\lambda(D) \geq 1$. We use $(u, v)$ denoting an arc with tail $u$ and head $v$. For $X, Y \subseteq V(D)$, we define

$$
\begin{aligned}
& (X, Y)_{D}=\{(x, y) \in A(D): x \in X, y \in Y\} ; \\
& \partial_{D}^{+}(X)=(X, V(D)-X)_{D} .
\end{aligned}
$$

Let

$$
\begin{aligned}
& d_{D}^{+}(X)=\left|\partial_{D}^{+}(X)\right| \\
& d_{D}^{-}(X)=\left|\partial_{D}^{-}(X)\right|
\end{aligned}
$$

When $X=\{v\}$, we write $d_{D}^{+}(v)=\left|\partial_{D}^{+}\{v\}\right|$ and $d_{D}^{-}(v)=\left|\partial_{D}^{-}\{v\}\right|$. Let $N_{D}^{+}(v)=\{u \in V(D)-v:(v, u) \in A(D)\}$ and $N_{D}^{-}(v)=\{u \in$ $V(D)-v:(u, v) \in A(D)\}$ denote the out-neighbourhood and in-neighbourhood of $v$ in $D$, respectively. We call the vertices in $N_{D}^{+}(v)$ and $N_{D}^{-}(v)$ the out-neighbours and the in-neighbours of $v$. Thus, for a digraph $D, \lambda(D) \geq 1$ if and only if, for any proper nonempty subset $\emptyset \neq X \subset V(D),\left|\partial_{D}^{+}(X)\right| \geq 1$. 
A dicycle cover of a digraph $D$ is a collection $\mathscr{C}$ of dicycles of $D$ such that $\bigcup_{C \in \mathscr{C}} A(C)=A(D)$. If $D$ is obtained from a simple undirected graph $G$ by assigning an orientation to the edges of $G$, then $D$ is an oriented graph. The main purpose is to investigate the number of dicycles needed to cover a Hamiltonian oriented graph. We prove the following.

Theorem 1. Let $D$ be an oriented graph on $n$ vertices and $m$ arcs. If $D$ has a Hamiltonian dicycle, then $D$ has a dicycle cover $\mathscr{C}$ with $|\mathscr{C}| \leq m-n+1$. This bound is best possible.

In the next section, we will first show that every Hamiltonian oriented graph with $n$ vertices and $m$ arcs can be covered by at most $m-n+1$ dicycles. Then we show that, for every Hamiltonian graph $G$ with $n$ vertices and $m$ edges, there exists an orientation $D=D(G)$ of $G$ such that any dicycle cover of $D$ must have at least $m-n+1$ dicycles.

\section{Proof of the Main Result}

In this section, all graphs are assumed to be simple. We start with an observation, stated as lemma below. A digraph $D$ is weakly connected if the underlying graph of $D$ is connected.

Lemma 2. A weakly connected digraph $D$ has a dicycle cover if and only if $\lambda(D) \geq 1$.

Proof. Suppose that $D$ has a dicycle cover $\mathscr{C}$. If $D$ is not strong, then there exists a proper nonempty subset $\varnothing \neq X \subset$ $V(D)$ such that $\left|\partial_{D}^{+}(X)\right|=0$. Since $D$ is weakly connected, $D$ contains an $\operatorname{arc}(u, v) \in(V(D)-X), X)_{D}$. Since $\mathscr{C}$ is a dicycle cover of $D$, there exists a dicycle $C \in \mathscr{C}$ with $(u, v) \in A(C)$. Since $(u, v) \in(V(D)-X), X)_{D}$, we conclude that $\varnothing \neq A(C) \cap(X, V(D)-X))_{D} \subseteq \partial_{D}^{+}(X)$, contrary to the assumption that $\left|\partial_{D}^{+}(X)\right|=0$. This proves that $D$ must be strong.

Conversely, assume that $D$ is strong. For any $\operatorname{arc} a=$ $(u, v) \in A(D)$, since $D$ is strong, there must be a directed $(v, u)$-path $P$ in $D$. It follows that $C_{a}=P+a$ is a dicycle of $D$ containing $a$, and so $\left\{C_{a}: a \in A(D)\right\}$ is a dicycle cover of D.

Let $C$ be a dicycle and let $a=(u, v)$ be an arc not in $A(C)$ but with $u, v \in V(C)$. Then $C+a$ contains a unique dicycle $C_{a}$ containing $a$. In the following, we call $C_{a}$ the fundamental dicycle of $a$ with respect to $C$.

Lemma 3. Let $D$ be an oriented graph on $n$ vertices and $m$ arcs. If $D$ has a Hamiltonian dicycle, then $D$ has a dicycle cover $\mathscr{C}$ with $|\mathscr{C}| \leq m-n+1$.

Proof. Let $C_{0}$ denote the directed Hamiltonian cycle of $D$. For each $a \in A(D)-A(C)$, let $C_{a}$ denote the fundamental dicycle of $a$ with respect to $C$. Then $\mathscr{C}=\left\{C_{0}\right\} \cup\left\{C_{a}: a \in A(D)-A(C)\right\}$ is a dicycle cover of $D$ with $|\mathscr{C}| \leq m-n+1$.

To prove that Theorem 1 is best possible, we need to construct, for each integer $n \geq 4$, a Hamiltonian oriented graph on $n$ vertices and $m$ arcs $D$ such that any dicycle cover $\mathscr{C}$ of $D$ must have at least $m-n+1$ dicycles in $\mathscr{C}$.
Let $G$ be a Hamiltonian simple graph. We present a construction of such an orientation $D=D(G)$. Since $G$ is Hamiltonian, we may assume that $V(G)=\left\{v_{1}, v_{2}, \ldots, v_{n}\right\}$ and $C=v_{1} v_{2}, \ldots, v_{n} v_{1}$ is a Hamiltonian cycle of $G$.

Definition 4. One defines an orientation $D=D(G)$ as follows.

(i) Orient the edges in the Hamiltonian cycle $C=$ $v_{1} v_{2}, \ldots, v_{n} v_{1}$ as follows:

$$
\left(v_{i+1}, v_{i}\right) \in A(D) \text {, }
$$

$$
i=1,2, \ldots, n-1,\left(v_{1}, v_{n}\right) \in A(D) .
$$

(ii) For each $i=2,3, \ldots, n-2$, and for each $j=i+2, i+$ $3, \ldots, n$, assign directions to edges of $G$ not in $E(C)$ as follows:

$$
\begin{aligned}
& \left(v_{i}, v_{j}\right) \in A(D), \\
& \quad \text { if } v_{i} v_{j} \in E(G)-E(C), i+1<j \leq n, \\
& \left(v_{1}, v_{j}\right) \in A(D), \\
& \quad \text { if } v_{1} v_{j} \in E(G)-E(C), i+1<j \leq n-1 .
\end{aligned}
$$
below.

We make the following observations stated in the lemma

Lemma 5. Each of the following holds for the digraph $D$ :

(i) The dicycle $C_{0}=v_{1} v_{n} v_{n-1}, \ldots, v_{3} v_{2} v_{1}$ is a Hamiltonian dicycle of $D$.

(ii) The digraph $D-A\left(C_{0}\right)$ is acyclic.

(iii) $N_{D}^{+}\left(v_{n}\right)=\left\{v_{n-1}\right\} ; N_{D}^{-}\left(v_{1}\right)=\left\{v_{2}\right\} ; N_{D}^{-}\left(v_{2}\right)=\left\{v_{3}\right\}$.

(iv) The dicycle $C_{0}$ is the only dicycle of $D$ containing the arc $\left(v_{1}, v_{n}\right)$.

(v) The dicycle $C_{0}$ is the unique Hamiltonian dicycle of $D$.

(vi) If $C^{\prime \prime}$ is a dicycle of $D$, then $C^{\prime \prime}$ contains at most one arc in $A(D)-A\left(C_{0}\right)$.

Proof. (i) follows immediately from Definition 4(i).

(ii) By Definition 4, the labels of the vertices $V(D)=$ $\left\{v_{1}, v_{2}, \ldots, v_{n}\right\}$ satisfy $\left(v_{i}, v_{j}\right) \in A(D)-A\left(C_{0}\right)$ only if $i<j$. It follows (e.g., Section 2.1 of [2]) that $D-A\left(C_{0}\right)$ is acyclic, and so (ii) holds.

(iii) This follows immediately from Definition 4

(iv) Let $C^{\prime}$ be a dicycle of $D$ with $\left(v_{1}, v_{n}\right) \in A\left(C^{\prime}\right)$. Since $\left(v_{1}, v_{n}\right) \in A\left(C^{\prime}\right) \cap A\left(C_{0}\right)$, we choose the largest label $i \leq n$, such that $\left(v_{1}, v_{n}\right),\left(v_{n}, v_{n-1}\right), \ldots,\left(v_{\mathrm{i}+1}, v_{i}\right) \in A\left(C^{\prime}\right) \cap A\left(C_{0}\right)$. Since $C^{\prime} \neq C_{0}$, we have $i \geq 3$. Since $C^{\prime}$ is a dicycle, there must be a vertex $v_{j} \in V(D)$ such that $\left(v_{i}, v_{j}\right) \in A\left(C^{\prime}\right)$. By the choice of $i$, we must have $\left(v_{i}, v_{j}\right) \notin A\left(C_{0}\right)$, and so $\left(v_{i}, v_{j}\right) \in$ $A(D)-A\left(C_{0}\right)$. By Definition 4(ii), we have $i+2 \leq j \leq n$, contrary to the fact that $C^{\prime}$ is a dicycle of $D$ containing $\left(v_{1}, v_{n}\right)$. This proves (iv).

(v) Let $C^{\prime}$ be a Hamiltonian dicycle of $D$. Since $V\left(C^{\prime}\right)=V(D)$, we have $v_{n} \in V\left(C^{\prime}\right)$. We claim that 
$\left(v_{1}, v_{n}\right) \in A\left(C^{\prime}\right)$. If $\left(v_{1}, v_{n}\right) \notin A\left(C^{\prime}\right)$, then there exists $v_{i} \in$ $V(C)\left(i \in\left\{v_{2}, v_{3}, \ldots, v_{n-1}\right\}\right)$ such that $\left(v_{i}, v_{n}\right) \in A\left(C^{\prime}\right)$. Hence, $\left(v_{i}, v_{n}\right),\left(v_{n}, v_{n-1}\right), \ldots,\left(v_{i+2}, v_{i+1}\right) \in A\left(C^{\prime}\right)$. By Definition 4(i) and (ii), $N^{+}\left(v_{i+1}\right) \subset\left\{v_{i+2}, v_{i+3}, \ldots, v_{n}\right\}$, contrary to the fact that $C^{\prime}$ is a Hamiltonian dicycle of $D$. Thus, $\left(v_{1}, v_{n}\right) \in A\left(C^{\prime}\right)$. It follows from Lemma 5 (iv) that we must have $C^{\prime}=C_{0}$.

(vi) By contradiction, we assume that $D$ has a dicycle $C^{\prime \prime}$ which contains two arcs: $a_{1}, a_{2} \in A(D)-A\left(C_{0}\right)$. Since $V(D)=\left\{v_{1}, v_{2}, \ldots, v_{n}\right\}$, we assume that $a_{1}=\left(v_{i}, v_{i^{\prime}}\right)$ and $a_{2}=\left(v_{j}, v_{j^{\prime}}\right)$. Without loss of generality and by Lemma 2 , we further assume that $1 \leq i<j<n$.

Let $i \geq t \geq 1$ be the smallest integer such that $v_{t} \in V\left(C^{\prime \prime}\right)$. Since $C^{\prime \prime}$ is a dicycle of $D$, there must be $v_{s} \in V\left(C^{\prime \prime}\right)$ such that $\left(v_{s}, v_{t}\right) \in A\left(C^{\prime \prime}\right)$. By Definition 4, either $\left(v_{s}, v_{t}\right) \in A\left(C_{0}\right)$ and $s=t+1<j$ or $\left(v_{s}, v_{t}\right) \in A(D)-A\left(C_{0}\right)$ and $1<s+$ $1<t$. By the choice of $t$, we can only have $s=t+1$ and $\left(v_{t+1}, v_{t}\right) \in A\left(C^{\prime \prime}\right) \cap A\left(C_{0}\right)$. Choose the largest integer $h$ with $t+1 \leq h<j$ such that $\left(v_{t+1}, v_{t}\right),\left(v_{t+2}, v_{t+1}\right), \ldots,\left(v_{h}, v_{h-1}\right) \in$ $A\left(C^{\prime \prime}\right) \cap A\left(C_{0}\right)$. Since $C^{\prime \prime}$ is a dicycle, there must be $v_{k}$ with $1 \leq k \leq n$ such that $\left(v_{k}, v_{h}\right) \in A\left(C^{\prime \prime}\right)$. By the maximality of $h$ and by Definition $4(\mathrm{i})$, we conclude that $\left(v_{k}, v_{h}\right) \notin A\left(C_{0}\right)$. By Definition 4(ii), $1 \leq k \leq h-2$. By the minimality of $t$, we must have $t \leq k \leq h-2$. It follows by $j>h$ that $C^{\prime \prime}$ cannot contain $a_{2}=\left(v_{j}, v_{j^{\prime}}\right)$, contrary to the assumption. This contradiction justifies (vi). lemma.

To complete the proof of Theorem 1, we present the next

Lemma 6. Let $G$ be a Hamiltonian simple graph. There exists an orientation $D=D(G)$ such that every dicycle cover of $D$ must have at least $m-n+1$ dicycles.

Proof. Let $G$ be a Hamiltonian graph and let $D=D(G)$ be the orientation of $G$ given in Definition 4. For notational convenience, we adopt the notations in Definition 4 and denote $V(D)=\left\{v_{1}, v_{2}, \ldots, v_{n}\right\}$. Thus, by Lemma $5(\mathrm{v}), C_{0}=$ $v_{1} v_{n} v_{n-1}, \ldots, v_{2} v_{1}$ is the unique Hamiltonian dicycle of $D$.

Let $\mathscr{C}$ be a dicycle cover of $D$. By Lemma 5(iv), we must have $C_{0} \in \mathscr{C}$. For each arc $a \in A(D)-A\left(C_{0}\right)$, since $\mathscr{C}$ is a dicycle cover of $D$, there must be a dicycle $C(a) \in \mathscr{C}$ such that $a \in A(C(a))$. By Lemma 5(vi), $A(C(a)) \cap A(D)-A\left(C_{0}\right)=\{a\}$. It follows that if $a, a^{\prime} \in A(D)-A\left(C_{0}\right)$, then $a \neq a^{\prime}$ implies $C(a) \neq C\left(a^{\prime}\right)$ in $\mathscr{C}$. Thus we have $\left\{C(a) \mid a \in A(D)-A\left(C_{0}\right)\right\} \subseteq$ $\mathscr{C}$. Hence

$$
\begin{aligned}
|\mathscr{C}| & \geq\left|\left\{C(a): a \in A(D)-A\left(C_{0}\right)\right\} \cup\left\{C_{0}\right\}\right| \\
& =m-n+1 .
\end{aligned}
$$

This proves the lemma.

By Lemmas 3 and 6, Theorem 1 follows. We are about to show that Theorem 1 can be applied to obtain dicycle cover bounds for certain families of oriented graphs. Let $T_{n}$ denote a tournament of order $n$. Then $T_{n}$ is an oriented graph. Camion $[13,14]$ proved that every strong tournament is Hamiltonian. Hence the corollary below follows from Theorem 1.
Corollary 7. Every strong tournament on $n$ vertices has a dicycle cover $\mathscr{C}$ with $|\mathscr{C}| \leq n(n-1) / 2-n+1$. This bound is best possible.

A bipartite graph $G(A, B)$ with vertex bipartition $(A, B)$ is balanced if $|A|=|B|$. If bipartite graph $G(A, B)$ has a Hamiltonian cycle, then $G$ is balanced. Let $K_{m, n}$ be a complete bipartite graph with vertex bipartition $(A, B)$ and $|A|=$ $m,|B|=n$; then $K_{m, n}$ has Hamiltonian cycle if and only if $m=n \geq 2$; that is, $K_{m, n}$ is balanced. Let $K_{n, n}$ denote a balanced complete bipartite graph.

Corollary 8. Every Hamiltonian orientation of balanced complete bipartite graph $K_{n, n}$ has a dicycle cover $\mathscr{C}$ with $|\mathscr{C}| \leq$ $(n-1)^{2}$. This bound is best possible.

Proof. Since an oriented balanced complete bipartite graph $K_{n, n}$ has $n^{2}$ arcs, so, by Theorem 1, we have $|\mathscr{C}| \leq n^{2}-2 n+1=$ $(n-1)^{2}$.

To prove the bound is best possible, we need to construct, for each integer $n \geq 2$, a Hamiltonian oriented balanced complete bipartite graph on $2 n$ vertices such that any dicycle cover $\mathscr{C}$ of $K_{n, n}$ must have at least $(n-1)^{2}$ dicycles in $\mathscr{C}$. We may assume that $V\left(K_{n, n}\right)=\left\{u_{1}, u_{2}, \ldots, u_{n}, v_{1}, v_{2}, \ldots, v_{n}\right\}$ and $C=u_{1} v_{1} u_{2} v_{2}, \ldots, u_{n} v_{n} u_{1}$ is a Hamiltonian cycle of $K_{n, n}$. We construct an orientation $D_{n, n}=D\left(K_{n, n}\right)$ as the orientation of Definition 4; thus, by Lemmas 5 and 6 , every dicycle cover $\mathscr{C}$ of $D_{n, n}$ must have at least $(n-1)^{2}$ dicycles. This proves the corollary.

\section{Dicycle Covers of 2 Sums of Digraphs}

In this section, we will show that Theorem 1 can also be applied to certain non-Hamiltonian digraphs which can be built via 2 sums. We start with 2 sums of digraphs.

Definition 9. Let $D_{n_{1}}=\left(V\left(D_{n_{1}}\right), A\left(D_{n_{1}}\right)\right)$ and $D_{n_{2}}=\left(V\left(D_{n_{2}}\right)\right.$, $\left.A\left(D_{n_{2}}\right)\right)$ be two disjoint digraphs; $a_{1}=\left(v_{12}, v_{11}\right) \in A\left(D_{n_{1}}\right)$ and $a_{2}=\left(v_{22}, v_{21}\right) \in A\left(D_{n_{2}}\right)$. The 2-sum $D_{n_{1}} \oplus_{2} D_{n_{2}}$ of $D_{n_{1}}$ and $D_{n_{2}}$ is obtained from the union of $D_{n_{1}}$ and $D_{n_{2}}$ by identifying the $\operatorname{arcs} a_{1}$ and $a_{2}$; that is, $v_{11}=v_{21}$ and $v_{12}=v_{22}$.

Definition 10. Let $D_{n_{1}}, D_{n_{2}}, \ldots, D_{n_{s}}$ be $s$ disjoint digraphs with $n_{1}, n_{2}, \ldots, n_{s}$ vertices, respectively. Let $D_{n_{1}} \oplus_{2} D_{n_{2}} \oplus_{2} \cdots \oplus_{2} D_{n_{s}}$ denote a sequence of 2 sums of $D_{n_{1}}, D_{n_{2}}, \ldots, D_{n_{s}}$, that is, $\left(\left(\left(D_{n_{1}} \oplus_{2} D_{n_{2}}\right) \oplus_{2} D_{n_{3}}\right) \oplus_{2} \cdots\right) \oplus_{2} D_{n_{s}}$.

Theorem 11. Let $D_{n_{1}}, D_{n_{2}}, \ldots, D_{n_{s}}$ be s disjoint Hamiltonian oriented graphs on $n_{1}, n_{2}, \ldots, n_{s}$ vertices and $m_{1}, m_{2}, \ldots, m_{s}$ arcs, respectively, and let $D=D_{n_{1}} \oplus_{2} D_{n_{2}} \oplus_{2} \cdots \oplus_{2} D_{n_{s}}$. Then $D$ has a dicycle cover $\mathscr{C}$ with $|\mathscr{C}| \leq|A(D)|-|V(D)|+1$. This bound is best possible.

Proof. By Theorem 1, $D_{n_{i}}(i=1,2, \ldots, s)$ has a dicycle cover $\mathscr{C}_{i}$ with $\left|\mathscr{C}_{i}\right| \leq m_{i}-n_{i}+1$. Let $\mathscr{C}=\bigcup_{i=1}^{s} \mathscr{C}_{i}$. Then $|\mathscr{C}| \leq$ $\left(m_{1}-n_{1}+1\right)+\left(m_{2}-n_{2}+1\right)+\cdots+\left(m_{s}-n_{s}+1\right)=\left(m_{1}+m_{2}+\right.$ $\left.\cdots+m_{s}\right)-\left(n_{1}+n_{2}+\cdots+n_{s}\right)+s=\left(m_{1}+m_{2}+\cdots+m_{s}-(s-\right.$ 1) $)-\left(n_{1}+n_{2}+\cdots+n_{s}-2(s-1)\right)+1=|A(D)|-|V(D)|+1$. By Definition $10, \mathscr{C}$ is a dicycle cover of $D$. Thus, $D$ has a dicycle cover $\mathscr{C}$ with $|\mathscr{C}| \leq|A(D)|-|V(D)|+1$. 
Let $G_{n_{i}}$ be $s$ disjoint Hamiltonian simple graphs for $i \epsilon$ $\{1,2, \ldots, s\}$. We may assume that $V\left(G_{n_{i}}\right)=\left\{v_{i 1}, v_{i 2}, \ldots, v_{i n_{i}}\right\}$ and $C_{i}=v_{i 1} v_{i 2}, \ldots, v_{i i_{i}} v_{i 1}$ is a Hamiltonian cycle of $G_{n_{i}}$, and let

$$
\begin{aligned}
& D_{n_{i}} \\
& =D\left(G_{n_{i}}\right) \text { be the orientation of } G_{n_{i}} \text { given in Definition } 4 .
\end{aligned}
$$

For notational convenience, we adopt the notations in Definition 4 and denote $V\left(D_{n_{i}}\right)=\left\{v_{i 1}, v_{i 2}, \ldots, v_{i n_{i}}\right\}$. Thus, by Lemma $5(\mathrm{v}), C_{i_{0}}=v_{i 1} v_{i n_{i}}, \ldots, v_{i 2} v_{i 1}$ is the unique Hamiltonian dicycle of $D_{n_{i}}$. Let $a_{i}=\left(v_{i 2}, v_{i 1}\right)$ be an arc of $D_{n_{i}}$. We construct the 2-sum digraph $D_{n_{1}} \oplus_{2} D_{n_{2}} \oplus_{2} \cdots \oplus_{2} D_{n_{s}}$ from the union of $D_{n_{1}}, D_{n_{2}}, \ldots, D_{n_{s}}$ by identifying the $\operatorname{arcs} a_{1}, a_{2}, \ldots, a_{s}$ such that $v_{11}=v_{21}=\cdots=v_{s 1}$ and $v_{12}=v_{22}=\cdots=v_{s 2}$. We assume that $v_{1}:=v_{11}=v_{21}=\cdots=v_{s 1}$ and $v_{2}:=v_{12}=v_{22}=$ $\cdots=v_{s 2}$ (the case when $s=2$ is depicted in Figure 1).

Claim 1. There does not exist a dicycle whose arcs intersect arcs in two or more $D_{n_{i}}$ 's $(i=1,2, \ldots, s)$.

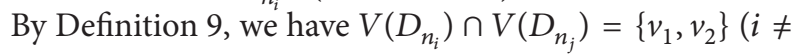
$j)$. Without loss of generality, we consider oriented graphs $D_{n_{1}}$ and $D_{n_{2}}$; suppose that there exists a dicycle $C_{0}$ such that

$$
\begin{aligned}
& \left\{A\left(C_{0}\right)-\left(v_{2}, v_{1}\right)\right\} \cap A\left(D_{n_{1}}\right) \neq \varnothing, \\
& \left\{A\left(C_{0}\right)-\left(v_{2}, v_{1}\right)\right\} \cap A\left(D_{n_{2}}\right) \neq \varnothing .
\end{aligned}
$$

Thus, there must exist four different arcs

$$
\left\{\left(v_{1 i^{\prime}}, v_{1}\right),\left(v_{1}, v_{2 i^{\prime \prime}}\right),\left(v_{2 j^{\prime \prime}}, v_{2}\right),\left(v_{2}, v_{1 j^{\prime}}\right)\right\} \in A\left(C_{0}\right)
$$

with $\left(v_{1 i^{\prime}}, v_{1}\right),\left(v_{2}, v_{1 j^{\prime}}\right) \in A\left(D_{n_{1}}\right)$ and $\left(v_{1}, v_{2 i^{\prime \prime}}\right),\left(v_{2 j^{\prime \prime}}, v_{2}\right) \in$ $A\left(D_{n_{2}}\right)$, as shown in Figure 2, or four different arcs

$$
\left\{\left(v_{1 s^{\prime}}, v_{2}\right),\left(v_{2}, v_{2 s^{\prime \prime}}\right),\left(v_{2 k^{\prime \prime}}, v_{1}\right),\left(v_{1}, v_{1 k^{\prime}}\right)\right\} \in A\left(C_{0}\right)
$$

with $\left(v_{1 s^{\prime}}, v_{2}\right),\left(v_{1}, v_{1 k^{\prime}}\right) \in A\left(D_{n_{1}}\right)$ and $\left(v_{2}, v_{2 s^{\prime \prime}}\right),\left(v_{2 k^{\prime \prime}}, v_{1}\right) \in$ $A\left(D_{n_{2}}\right)$, as shown in Figure 3 .

By Definition 9, Lemma 5(iii), and (6), we have $N_{D}^{-}\left(v_{1}\right)=$ $\left\{v_{2}\right\}$, and so $v_{1 i^{\prime}}=v_{2}$ or $v_{2 k^{\prime \prime}}=v_{2}$, contrary to the assumption that $C_{0}$ is a dicycle. This proves Claim 1.

By Claim 1, for every dicycle $C$ in $D$, all arcs in $C$ (except for the arc $\left.\left(v_{2}, v_{1}\right)\right)$ belong to exactly one of oriented graphs $D_{n_{i}}(i=1,2, \ldots, n)$. By Definition 4 and Lemma 6, every dicycle cover of oriented graph $D_{n_{i}}(i=1,2, \ldots, n)$ must have at least $m_{i}-n_{i}+1$ dicycles. This completes the proof.

By Corollary 7 and Theorem 11, we have the following corollary.

Corollary 12. Let $D_{n_{1}}, D_{n_{2}}, \ldots, D_{n_{s}}$ be $s$ disjoint strong tournaments with $n_{1}, n_{2}, \ldots, n_{s}$ vertices, respectively. Then $D_{n_{1}} \oplus_{2} D_{n_{2}} \oplus_{2} \cdots \oplus_{2} D_{n_{s}}$ has a dicycle cover $\mathscr{C}$ with $|\mathscr{C}| \leq$ $\left(n_{1}\left(n_{1}-1\right) / 2+n_{2}\left(n_{2}-1\right) / 2+\cdots+n_{s}\left(n_{s}-1\right) / 2\right)-\left(n_{1}+n_{2}+\right.$ $\left.\cdots+n_{s}\right)+s$. This bound is best possible.

Let $G_{n}$ be a Hamiltonian graph with $n$ vertices and $m$ arcs; let $D_{n}^{i}$ ( $i$ is an integer) denote a Hamiltonian orientation of

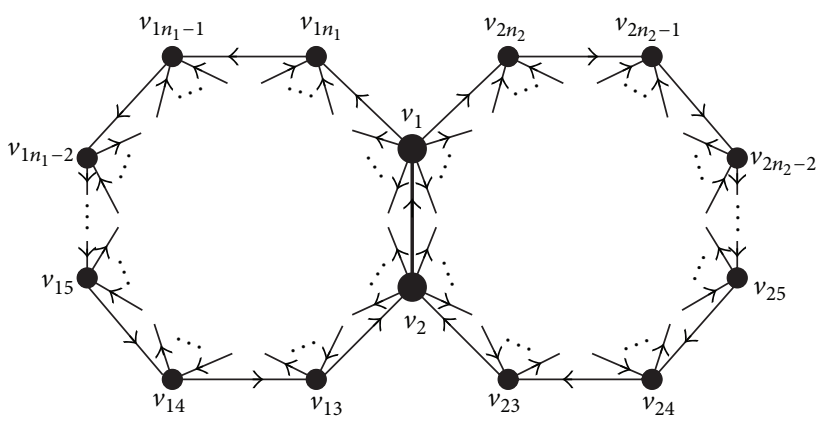

FIGURE 1: The 2-sum digraph for $D_{n_{1}}$ and $D_{n_{2}}$.

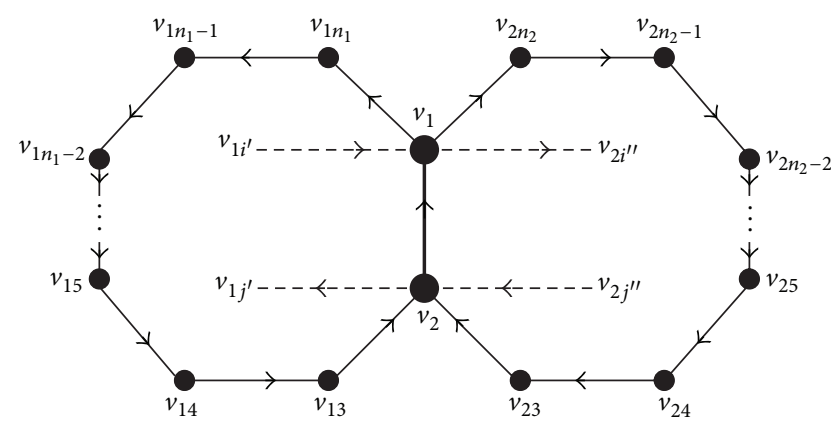

FIGURE 2

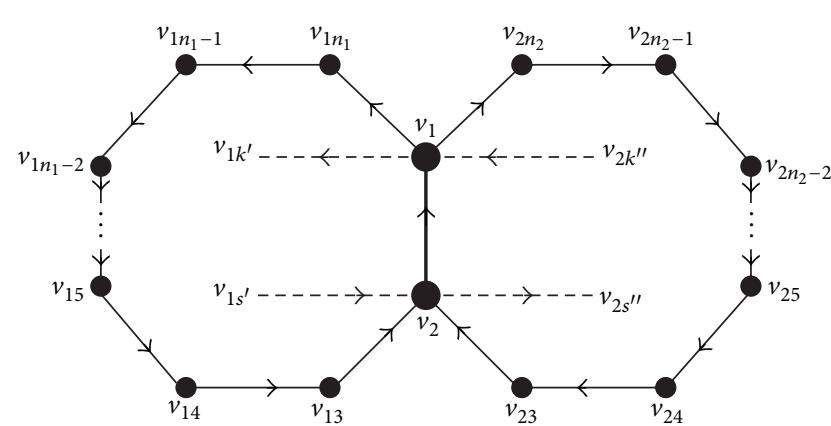

Figure 3

$G_{n}$. For a positive integer $s$, let $H\left(G_{n}, s\right)$ denote the family of all 2-sum generated digraphs $D_{n}^{1} \oplus_{2} D_{n}^{2} \oplus_{2} \cdots \oplus_{2} D_{n}^{s}$, as well as a member in the family (for notational convenience). By the definition of $H\left(G_{n}, s\right)$, we have $H\left(G_{n}, 1\right)=D_{n}^{1}$ and $H\left(G_{n}, s\right)=$ $H\left(G_{n}, s-1\right) \oplus_{2} D_{n}^{s}$. The conclusions of the next corollaries follow from Theorem 1 . The sharpness of these corollaries can be demonstrated using similar constructions displayed in Lemma 6 and Corollary 8.

Corollary 13. Let $m, n \geq 3$ be integer, let $G_{n}$ be a Hamiltonian graph with $n$ vertices and $m$ edges, and let $K_{n}$ be a complete graph on $n \geq 3$ vertices:

(i) Any member in $H\left(G_{n}, s\right)$ has a dicycle cover $\mathscr{C}$ with $|\mathscr{C}| \leq s(m-n+1)$. This bound is best possible.

(ii) In particular, any $H\left(K_{n}, s\right)$ has a dicycle cover $\mathscr{C}$ with $|\mathscr{C}| \leq s(n(n-1) / 2-n+1)$. This bound is best possible. 
Corollary 14. Let $m, n \geq 3$ be integer, let $B_{n}$ be a Hamiltonian bipartite graph with $2 n$ vertices and $m$ edges, and let $K_{n, n}$ be a complete bipartite graph:

(i) Any $H\left(B_{n}, s\right)$ has a dicycle cover $\mathscr{C}$ with $|\mathscr{C}| \leq s(m-$ $2 n+1)$. This bound is best possible.

(ii) In particular, any $H\left(K_{n, n}, s\right)$ has a dicycle cover $\mathscr{C}$ with $|\mathscr{C}| \leq s(n-1)^{2}$. This bound is best possible.

\section{Conflict of Interests}

The authors declare that there is no conflict of interests regarding the publication of this paper.

\section{References}

[1] J. A. Bondy and U. S. R. Murty, Graph Theory, vol. 244 of Graduate Texts in Mathematics, Springer, New York, NY, USA, 2008.

[2] J. Bang-Jensen and G. Gutin, Digraphs: Theory, Algorithms and Applications, Springer, 2nd edition, 2010.

[3] J. A. Bondy, "Small cycle double covers of graphs," in Cycles and Rays, pp. 21-40, Kluwer Academic Publishers, Dordrecht, The Netherlands, 1990.

[4] Y. X. Luo and R. S. Chen, "Cycle covers of 2-connected 3-regular graphs," Mathematics and Applications, vol. 9, supplement, pp. 23-25, 1996 (Chinese).

[5] H.-J. Lai, "Cycle covers of planar graphs," Congressus Numerantium, vol. 122, pp. 33-46, 1996.

[6] H.-J. Lai and H. Y. Lai, "Cycle covering of plane triangulations," Journal of Combinatorial Mathematics and Combinatorial Computing, vol. 10, pp. 3-21, 1991.

[7] H.-J. Lai and H. Y. Lai, "Cycle covers in graphs without subdivisions of K4," Journal of Combinatorial Mathematics and Combinatorial Computing, vol. 9, pp. 201-207, 1991.

[8] H.-J. Lai and H. Y. Lai, "Small cycle covers of planar graphs," Congressus Numerantium, vol. 85, pp. 203-209, 1991.

[9] D. W. Barnette, "Cycle covers of planar 3-connected graphs," Journal of Combinatorial Mathematics and Combinatorial Computing, vol. 20, pp. 245-253, 1996.

[10] G. Fan, "Subgraph coverings and edge switchings," Journal of Combinatorial Theory Series B, vol. 84, no. 1, pp. 54-83, 2002.

[11] H.-J. Lai and X. Li, "Small cycle cover of 2-connected cubic graphs," Discrete Mathematics, vol. 269, no. 1-3, pp. 295-302, 2003.

[12] F. Yang and X. Li, "Small cycle covers of 3-connected cubic graphs," Discrete Mathematics, vol. 311, no. 2-3, pp. 186-196, 2011.

[13] P. Camion, "Chemins et circuits hamiltoniens des graphes complets," Comptes Rendus de l'Académie des Sciences, vol. 249, pp. 2151-2152, 1959.

[14] J. W. Moon, “On subtournaments of a tournament," Canadian Mathematical Bulletin, vol. 9, pp. 297-301, 1966. 


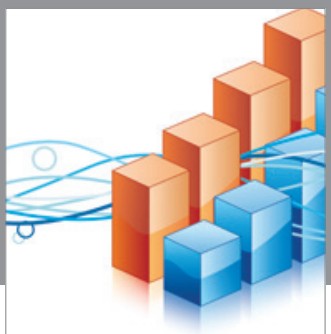

Advances in

Operations Research

vatem alat4

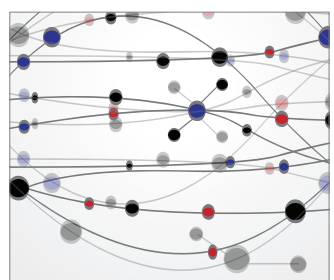

\section{The Scientific} World Journal
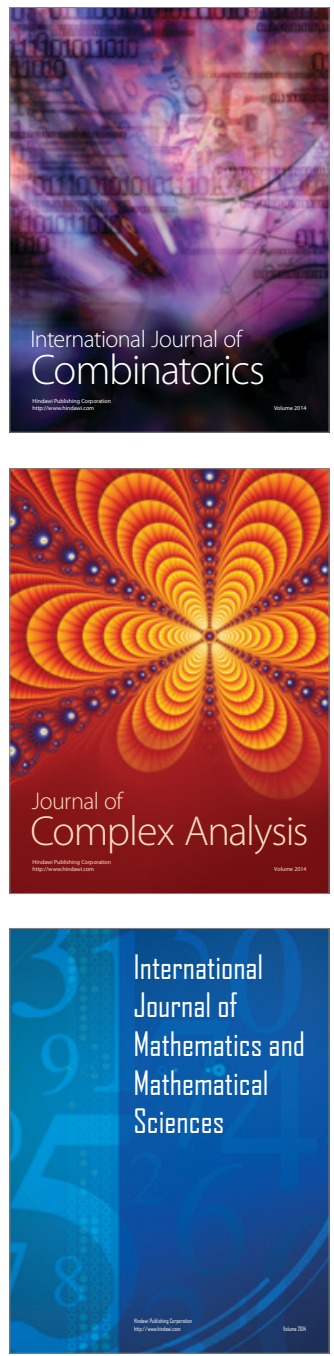
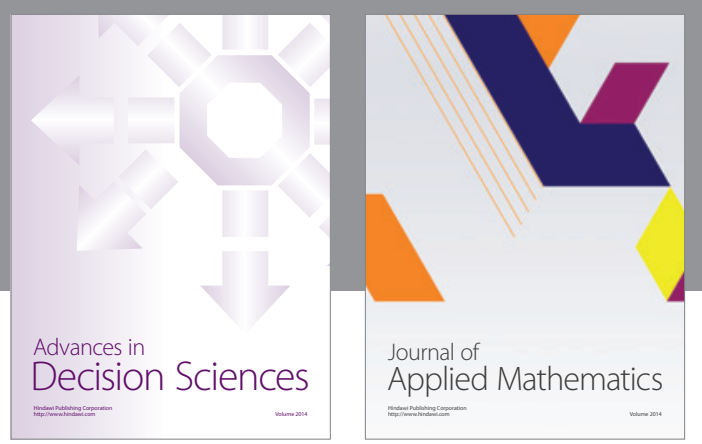

Algebra

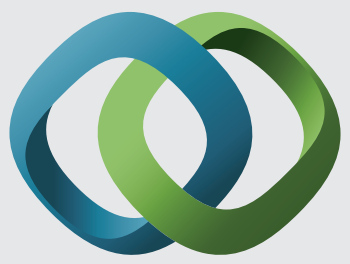

\section{Hindawi}

Submit your manuscripts at

http://www.hindawi.com
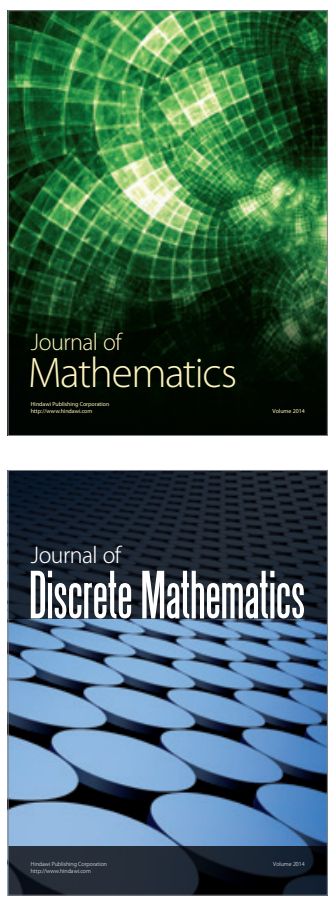

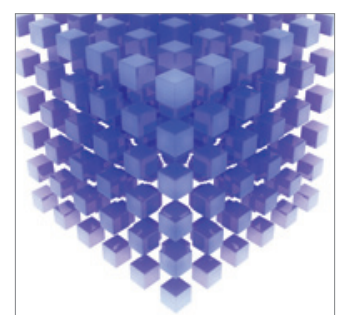

Mathematical Problems in Engineering
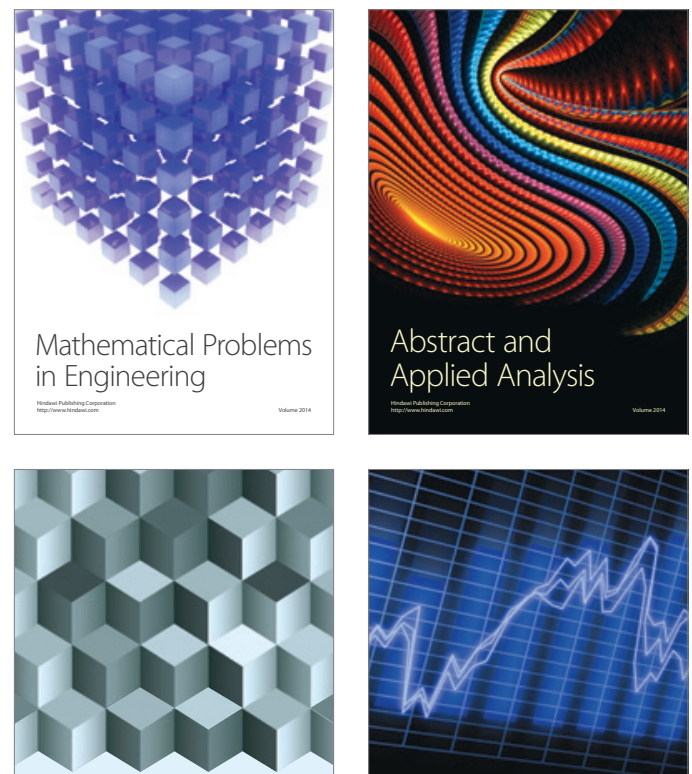

Journal of

Function Spaces

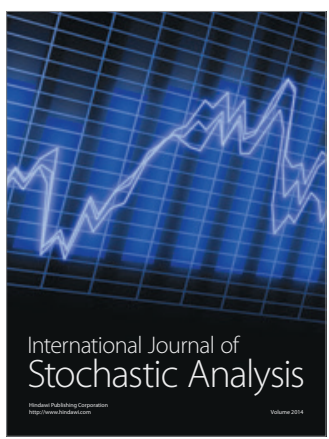

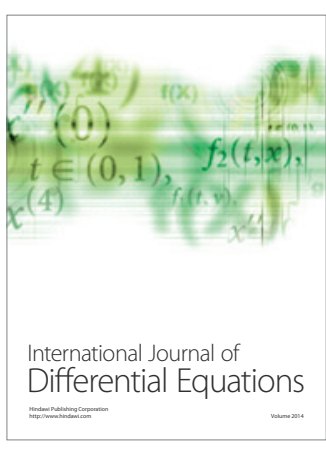
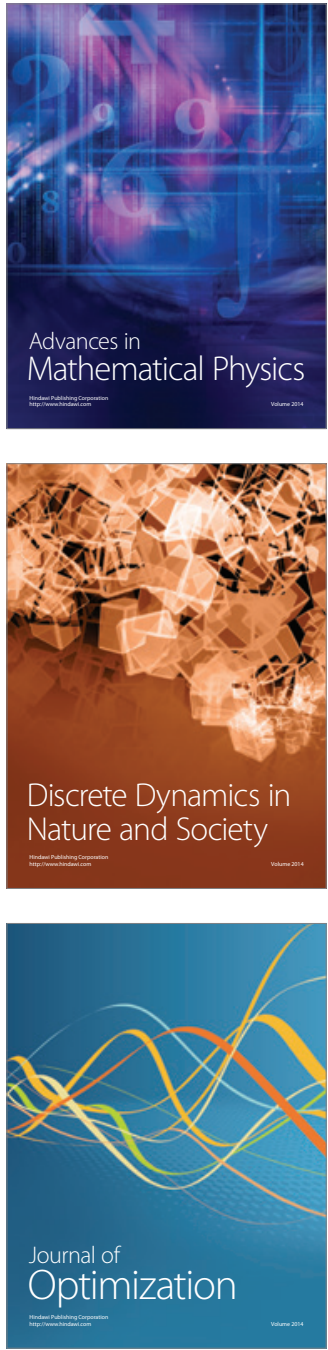